\title{
Independent subpatterns of sense organs require independent genes of the achaete-scute complex in Drosophila larvae
}

\author{
Christine Dambly-Chaudière and Alain Ghysen \\ Laboratoire de Génétique, Université Libre de Bruxelles, 1640 Rhode-St-Genése, Belgium
}

\begin{abstract}
The achaete-scute gene complex (AS-C) is a series of adjacent genes that play an essential role in the development of the Drosophila nervous system. A deletion of the entire complex results in the lack of all external sense organs and of most peripheral neurons in the larva. The analysis of reciprocal left-right deletions reveals that the larval pattern of sense organs results from the addition of several independent subpatterns, each of which specifically requires a particular set of AS-C functions. Two of the subpatterns point to concealed homologies that are confirmed by the analysis of bxd mutants. We conclude that AS-C defines the basic topology of the pattern, rather than the type or precise location of the elements.
\end{abstract}

[Key Words: Sensory system; pattern formation; Drosophila larva; neurogenesis; achaete-scute complex]

Received December 23, 1986; revised version received and accepted February 2, 1987.

How reproducible patterns are generated during the development of an organism remains an open question. In Drosophila, mutational analyses have identified several genes or gene complexes specifically involved in the formation of spatial patterns. One of them is the achaetescute gene complex (AS-C), which is required for the differentiation of sense organs at particular positions on the adult epidermis. Most mutations and rearrangements affecting this complex are recessive. Their phenotype, presumably corresponding to some loss of AS-C function, is a reduction or suppression of adult sense organs (Serebrovsky and Dubinin 1930). In addition, there are a few dominant mutations that have the opposite effect and cause the development of supernumerary sense organs, as a result of an excess of function of AS-C (Campuzano et al. 1986; García-Alonso and García-Bellido 1986). This suggests that AS-C is directly involved in the development decision by which an epithelial cell becomes committed to develop into a sense organ (Garcia-Bellido and Santamaria 1978).

Many AS-C mutations are rearrangements (Sutton 1943; Lindsley and Grell 1968) with breakpoints scattered over the entire complex (Carramolino et al. 1982). In some cases the two elements-left and right-of different rearrangements can be recombined, thereby generating a deficiency for the region between the two breakpoints (Muller and Prokofyeva 1935). In other rearrangements, where part of the complex is translocated to another chromosome, the two elements of the broken complex can be separately recovered and their function assessed (García-Bellido 1979). This genetic dissection of the complex has led to the identification of four adjacent functional units: achaete $(\mathrm{ac})$, scute $\alpha(\operatorname{sc} \alpha)$, lethal of scute (l'sc), and scute $\beta(\mathrm{sc} \beta)$ from left (distal) to right (proximal to the centromere). ac and sc $\alpha$ define two reciprocal sets of bristles on the adult: the lack of ac affects mostly the small bristles on the head and thorax, whereas the lack of $s c \alpha$ eliminates the large bristles of the head and thorax as well as all abdominal bristles. The absence of l'sc results in abnormal development of the embryonic central nervous system (CNS) (GarcíaBellido and Santamaria 1978; Jimenez and Campos-Ortega 1979; White 1980), and that of $\operatorname{sc} \beta$ causes an adult phenotype similar to but weaker than that of $s c \alpha$, possibly because $\operatorname{sc} \beta$ lesions alter the expression of $\operatorname{sc} \alpha$ (Campuzano et al. 1985). In addition, other genes to the right of sc $\beta$ are also required for the development of the CNS (Jimenez and Campos-Ortega 1979; White 1980). Thus, the AS-C genes are required for the development of two different systems, the adult sensory and the embryonic central, both of which involve the formation of precise patterns of neural elements. This raises the question of how these different genes combine their effects to achieve the development of a normal nervous system, and more specifically, whether AS- $\mathrm{C}$ functions are involved in defining cell types, or positions.

We have studied the function of AS-C in the formation of the larval pattern of sense organs, where both the type and the position of all elements are defined accurately (Campos-Ortega and Hartenstein 1985; DamblyChaudière and Ghysen 1986; Ghysen et al. 1986; Bodmer and Jan 1987). The larval sensory system comprises more than 250 external sense organs of three different types which occupy reproducible locations on the 
larval epidermis. The entire complement of peripheral neurons can be visualized by either anti-horseradish peroxidase (HRP) antibodies (Jan and Jan 1982), or by a monoclonal antibody, 21A4 (Ghysen et al. 1986); most sensory neurons can also be identified by their position and typical dendrite. The high resolution of this system allowed us to assess the effect of various AS-C deletions on each element of the entire pattern.

\section{Results}

\section{The normal pattern of sensory neurons}

This pattern has been described elsewhere (Campos-Ortega and Hartenstein 1985; Ghysen et al. 1986); we will summarize its major features. Three types of peripheral neurons can be recognized: (1) es neurons, which innervate the external sense organs; (2) ch neurons, which innervate the chordotonal sense organs; and (3) nd neurons, which later differentiate numerous dendrites and are probably proprioceptive. In addition, most segments contain a few neurons that innervate tracheal branches, and a few bipolar neurons of unknown function (Bodmer and Jan 1987). The complete pattern of peripheral neurons in an abdominal segment is illustrated in Figure 1a.

The sensory neurons are segmentally arranged according to the five patterns shown in Figure 2 (upper panel): $\mathrm{T} 1$, in the prothoracic segment; $\mathrm{T}$, in the mesoand metathoracic segments; $A$, repeated in the first seven abdominal segments; $A 8$, in the eighth abdominal segment; and A9 comprising the ninth and tenth abdominal neurons. Within most segments, the cell bodies are arranged in four clusters named $v, v^{\prime}, 1$, and $d$ from the most ventral to the most dorsal. The prothoracic segment contains an additional cluster, $\mathrm{v}^{\prime \prime}$; the clusters found in the A8 and A9 patterns are all dorsal. Each peripheral neuron is identified by the segmental pattern to which it belongs (T1, T, A, A8, A9), its cluster $\left(v, v^{\prime}, v^{\prime \prime}, 1\right.$, d), its nature (es, ch, nd), and its position within the cluster (A, B. . from ventral to dorsal). When two or three adjacent neurons join their dendrites and innervate a common sense organ, it is not possible to tell them apart and they are named according to the number of apposed dendrites (e.g., des2 are the two neurons that jointly innervate the diminutive dorsal hair, $\mathrm{h} 3$, in the abdominal segments).

\section{Requirement for AS-C in the embryo}

We first examined whether AS-C genes are required for the development of the larval sensory system. All
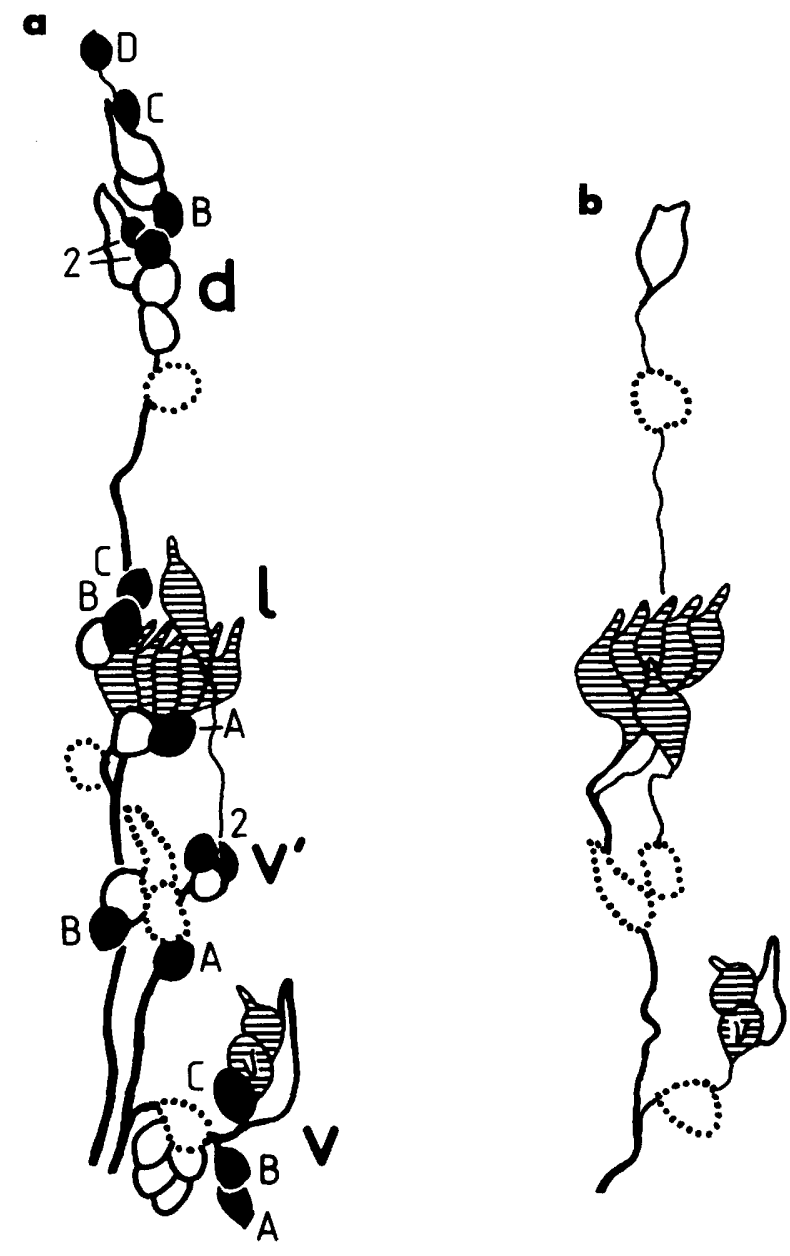

Figure 1. The peripheral neurons in an abdominal segment of normal $(a)$ and mutant $(b)$ Drosophila embryos. Peripheral neurons are of different types (see text): es neurons (black), ch neurons (striped), nd neurons (white), and tracheal and bipolar neurons (dotted outline). The dendrites of es and nd neurons have been omitted for clarity. In the wild type, the neurons are arranged in four clusters named, $v, v^{\prime}, l$, and $d$ from ventral to dorsal; within each cluster, individual es neurons are identified by a letter or a number (Ghysen et al. 1986). The Df260.1 pattern illustrated in $b$ is atypically well organized; this particular segment was selected to facilitate the comparison with the wild type. In most cases the pattern is more or less disrupted, massive axonal misroutings may occur, and some of the tracheal or bipolar neurons may be missing. These defects are more pronounced in larger deficiencies. Even in $D f B l d$, however, each segment retains at least three peripheral neurons /one of which is the dorsal bipolar neuron) in addition to the usually complete set of ch neurons.

Figure 2. The subpatterns of es neurons corresponding to the four classes $A, B, C$, and $D$ defined in Table 1. (Upper panel) Five segmental patterns observed in normal embryos and larvae (adapted from Ghysen et al. 1986 and Dambly-Chaudière and Ghysen 1986). spA-D: Spiracular sensory neurons. (Lower panels) For each class A-D, the sense organs that belong to that class and the dendrites of the innervating neurons. The dotted lines are a standard diagram of the clusters of neural cell bodies and axon bundles; no attempt was made to represent the actual size of the cell clusters in the different classes. In the case of the Keilin organs, the neurons are difficult to tell apart (see legend to Table 1), and the allocation of specific neurons to classes $\mathrm{A}, \mathrm{C}$, and $\mathrm{D}$ is tentative. In $A$ and $C$, the arrows point to doubly innervated abdominal structures, respectively, the papilla p6 $(A)$ and the diminutive hair h3 $(C)$, that are transformed in bxd mutants, as shown in Figs. 4 and 5. 

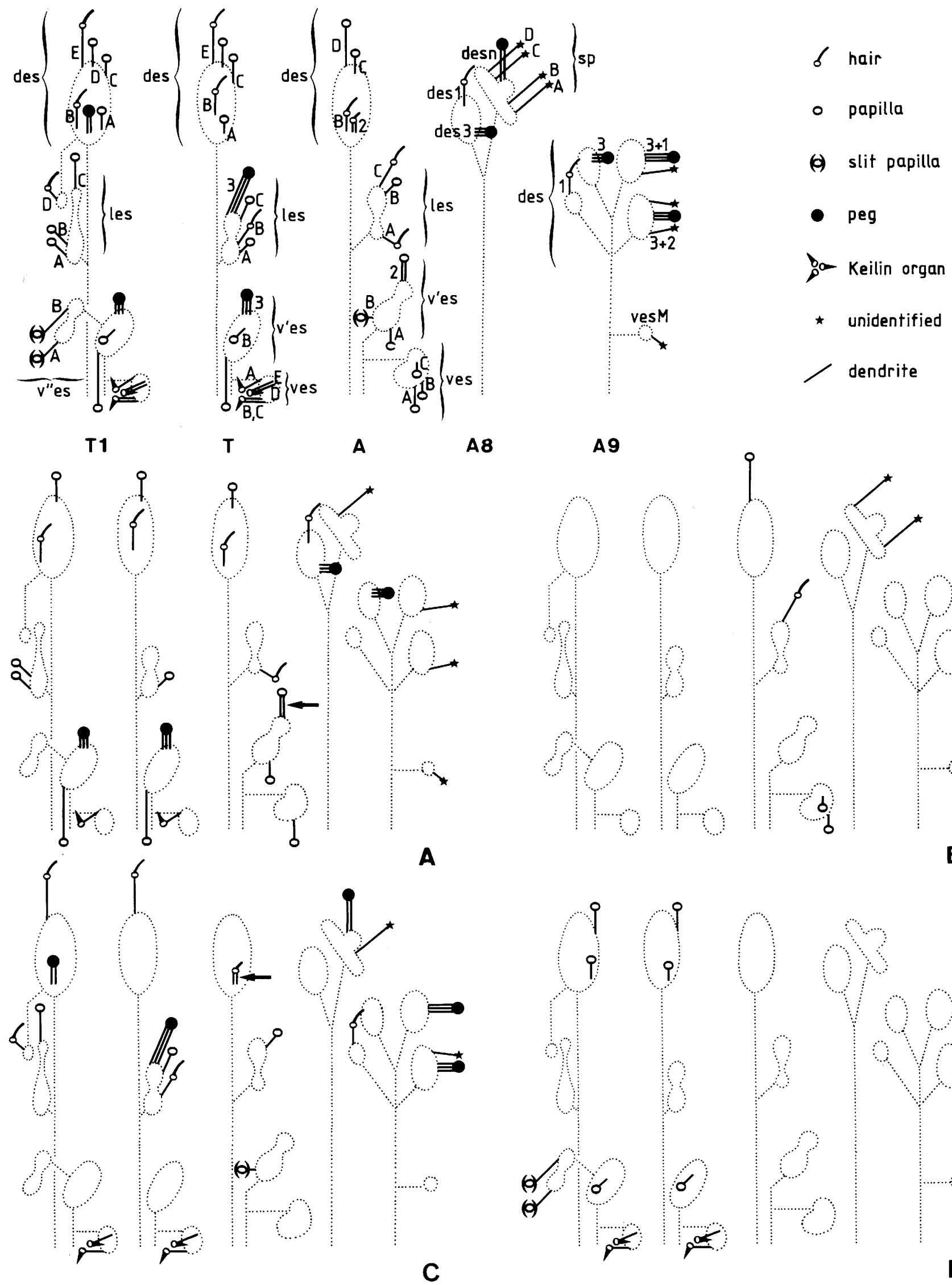

A8
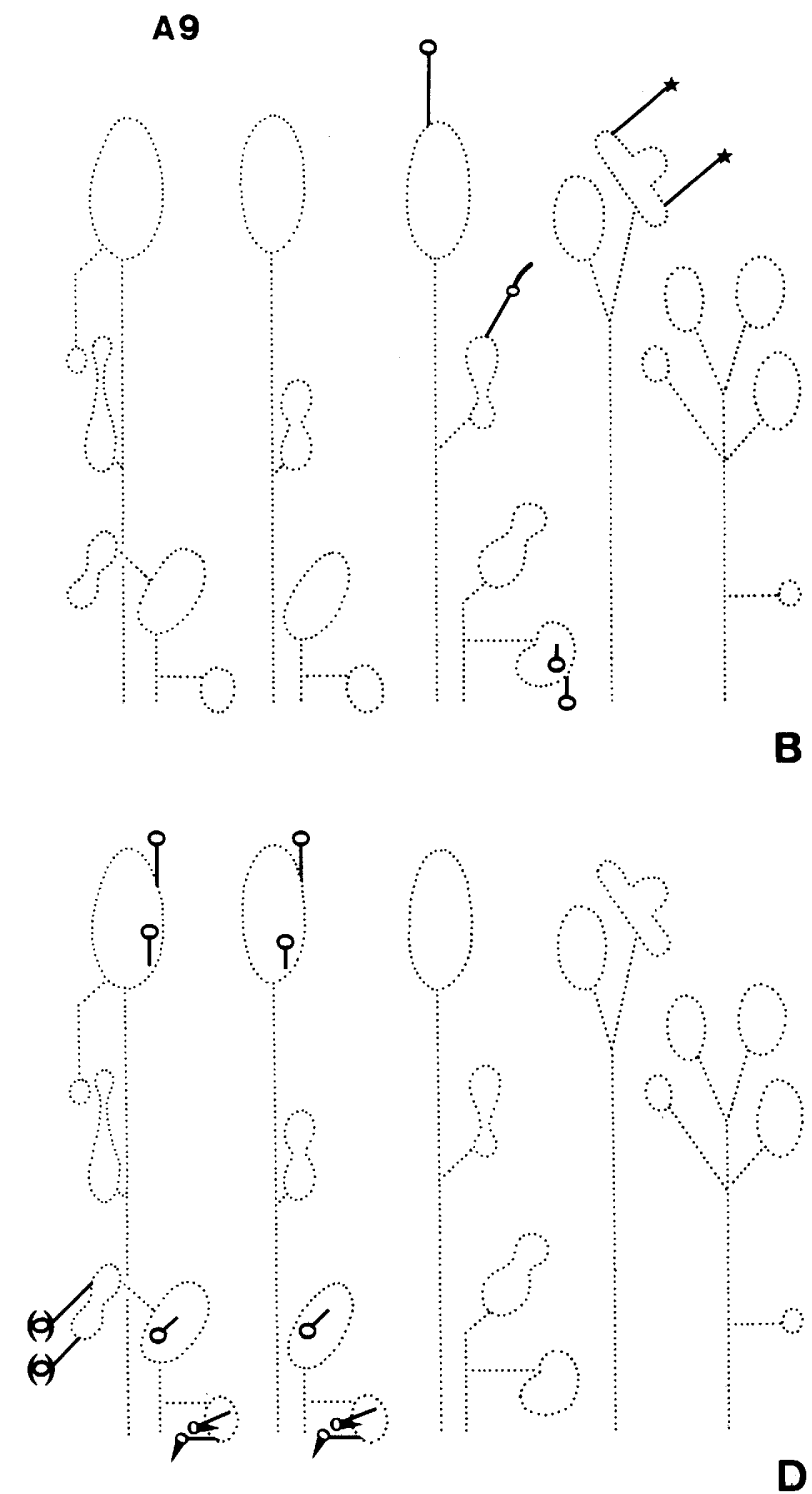

B

Figure 2. (See facing page for legend.) 
known AS-C regions are deleted in $D f 260.1$, a terminal deficiency of the first chromosome (Lindsley and Grell 1968). The resulting phenotype is illustrated in Figure 1b in the case of the A pattern. All es neurons and most of the nd neurons are removed; the ch neurons are not affected. The lack of effect on ch neurons could indicate that some elements of AS-C are still present in Df260.1. We analyzed two larger deficiencies, $D f s v r$ and $D f B l d$, the latter of which deletes more than 10 bands proximal to AS-C. The phenotypes of both deficiencies remain very similar to that of $D f 260.1$, except that the neurons tend to be more disorganized, mostly in the ventral region. Thus, we conclude that the AS-C system is an essential element of the development of es and nd neurons, but not of ch neurons. The following analysis will be restricted to the es neurons, each of which can be uniquely recognized on the basis of its position and typical dendrite (Ghysen et al. 1986). In addition, the complete pattern of external sense organs has been described, and the es neurons innervating each organ have been identified unambiguously (Dambly-Chaudière and Ghysen 1986), allowing us to rely on two independent descriptions of mutant phenotypes.

\section{Dissection of AS-C}

The classical way to assess the function of the different genes in the AS-C region is to use synthetic deficiencies that remove consecutive stretches of that region (Muller and Prokofyeva 1935). However, such synthetic deficiencies are usually derived from rearrangements that bring AS-C genes in contact with heterochromatin (Raffel and Muller 1940), resulting in variegation of nearby genes. Preliminary experiments indicated that the synthetic deficiencies classically used to define ac, sc $\alpha$, and l'sc (Muller and Prokofyeva 1935) show a strong inactivation of adjacent genes in the embryo, leading to inconsistent or incorrect assignments.

The present analysis relies mostly on the availability of rearrangements that split the complex into two reciprocal fragments that can be recovered separately (Garcia-Bellido 1979). If a sense organ is formed when either fragment is present, one can conclude that the two pieces express repetitive functions that can substitute for each other. If it is formed only when both fragments are present, this suggests that the two reciprocal pieces express different functions which are all required by this organ. Finally, if a given sense organ is formed whenever one fragment is present, and is missing when only the reciprocal fragment is present, one can conclude that the development of this organ specifically requires a function expressed by the first fragment.

\section{Subdivision of $A S-C$ by scute ${ }^{\mathrm{H}}$}

We split the AS-C into two pieces by means of the translocation $T(1,4) s c^{H}$, where the left part of the complex $\left(s c^{H L}\right)$ has moved to the fourth chromosome, while the right part $\left(s c^{H R}\right)$ has remained on the first chromosome (Fig. 3). Individuals that have $s c^{H L}$ on the fourth chromosome (and that are deleted for AS-C on the first) will contain the genes ac, sc $\alpha$, and l'sc but not $\operatorname{sc} \beta$, while individuals that have $s c^{H R}$ on the first chromosome will contain $\operatorname{sc} \beta$ and the genes to its right but not ac, $\operatorname{sc} \alpha$, nor $l^{\prime} s c$. The phenotypes resulting from the presence and absence of each fragment are presented in Table 1 (first two lines). In most cases, a given neuron strictly depends on one of the two fragments, that is, it will be completely absent when that fragment is missing and substantially present when that fragment is retained. Thus, most sensory neurons fall into two reciprocal classes: one depends entirely on $s c^{H L}$, and the other depends entirely on the reciprocal fragment, $s c^{H R}$. A third class comprises the seven $\mathrm{T} 1$ and five $\mathrm{T}$ neurons that are missing in both cases.

\section{Subdivision of $\mathrm{sc}^{\mathrm{HL}}$ by scute 8}

The $s c^{H L}$ part of AS-C contains two genes that are essential for the development of complementary sets of adult bristles, ac and sc $\alpha$, in addition to a gene that is not involved in bristle differentiation, 1 'sc. We used another rearrangement, $s c^{8}$, to subdivide this region further. $s c^{8}$ breaks AS-C between ac and sco, yielding two halves that can be recovered separately: $s c^{8 L}$, which contains only ac, and $s c^{8 R}$, which contains all other AS-C genes (Fig. 3). The phenotypes of embryos lacking either $s c^{8 L}$ or $s c^{8 R}$ are summarized in Table 1 (lines 3-4).

All neurons of the $s c^{H L}$ class are found in at least $60 \%$ of the cases in the presence of the $s c^{8 L}$ fragment (line 3), suggesting that the presence of achaete is sufficient for the development of this class of neurons. However, most of these neurons are also found, albeit at a lower frequency, in embryos where only $s c^{8 R}$ is present (line 4), implying that genes located to the right of $s c^{8}$ can to some extent palliate for the lack of ac. Since we have seen that the region to the right of $s c^{H}$ is unable to induce the formation of these neurons, it follows that the genes that can partly substitute for achaete are located between $s c^{8}$ and $s c^{H}$, that is, they are sc $\alpha$ and/or l'sc. We confirmed and refined this result by analyzing the external sense organs of larvae deleted for $s c \alpha\left(s c^{8 L} s c^{4 R}\right)$, deleted for ac $\left(a c^{1}\right)$, or deleted for both ac and $s c \alpha$ $\left(y^{3 P L} S^{S 1 R}\right)$, and of embryos deleted for all three genes ac, sc $\alpha$, and l'sc $\left(D f s c^{H}\right)$. In all cases only the sense organs innervated by neurons of the $s c^{H L}$ class are affected; these sense organs are found at an average frequency of 0.9 in $s c^{8 L} s c^{4 R}$, of 0.5 in $a c^{1}$, of 0.2 in $y^{3 P L} s c^{S 1 R}$, and of 0 in $D f s c^{H}$ embryos. Thus, thece of sc $\alpha$ has little if any

effect by itself, but markedly increases the lack of sense organs resulting from the absence of ac, confirming that sc $\alpha$ can to some extent substitute for ac. Furthermore, even in the absence of both ac and sc $\alpha$, many sense organs of this class are still present, albeit at a very low frequency, suggesting that l'sc can also substitute for ac, though rather inefficiently.

The seven $\mathrm{Tl}$ and five $\mathrm{T}$ neurons that are missing when either $s c^{H L}$ or $s c^{H R}$ are present depend entirely on the presence of the $s c^{8 R}$ fragment. As expected, all neurons that depend on the $s c^{H R}$ fragment are also found in the presence of $\mathrm{sc}^{8 R}$. 
Table 1. The phenotypes of partial deficiencies for AS-C

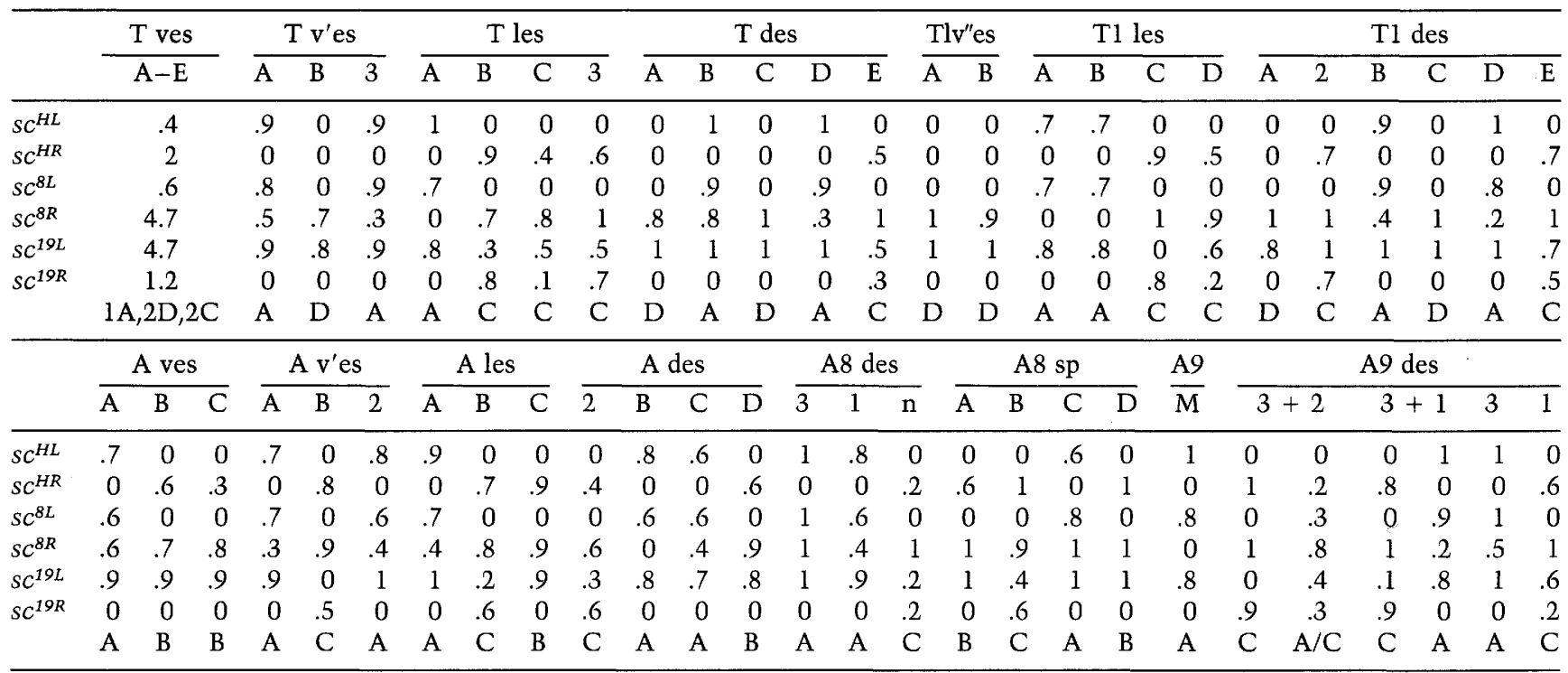

The upper line lists the es neurons in the T, T1, A, A8 and A9 patterns (see Fig. 2, upper panel). In T1 and T, the v and $\mathrm{v}^{\prime}$ clusters are identical, and therefore the data were pooled and given for $T$. The $v$ cluster contains five neurons, $A-E$, that innervate the Keilin organ: The proximity of their dendrites makes it difficult to decide which is which when only one or two are present, and therefore the five were considered together. The second to seventh lines give the frequency of each neuron in the presence of the fragment of AS-C mentioned in the leftmost column. In most cases the neuron is either entirely absent, or detected in more than half of the cases (see Experimental procedures). The genotypes are as follows: $\left.s c^{H L}, D f(1) 260.1 / Y ; D p(1 ; 4) s c^{H /}+; s c^{H R}, D f(1) s c^{H / Y} ; s c^{8 L}, D f(1) 260.1\right)$ $D p(1 ; Y) s c^{8} ; s c^{8 R}, D f(1) s c^{8} / Y ; s c^{19 L}, D f(1) 260.1 / Y ; D p(1 ; 2) s c^{19} /+; s c^{19 R}, D f(1) s c^{19} / Y$. Last line: assignment of each neuron to one of four classes, A, B, C, or D (see text).

\section{Subdivision of $\mathrm{sc}^{\mathrm{HR}}$ by scute ${ }^{19}$}

The right part of AS-C, present in the $s c^{H R}$ fragment, can be subdivided into two subregions by using another translocation, $T(1 ; 2) s c^{19}$ (Fig. 3). The subregion between the $s c^{H}$ and the $s c^{19}$ breakpoints corresponds to the $\operatorname{sc} \beta$ gene defined earlier (Garcia-Bellido 1979), while the subregion between the breakpoints of $s c^{19}$ and of $D f(1) 260.1$ defines a new AS-C element that we call sc $\gamma$. The phenotypes of embryos carrying either part of the $T(1 ; 2) S c^{19}$ translocation, $s c^{19 L}$ (including all AS-C genes except for sc $\gamma$, or $s c^{19 R}$ (containing only scy) are given in Table 1 (lines 5 and 6). Two behaviors are observed among the neurons of the $s c^{H R}$ class. Some neurons depend entirely on the presence of the $s c^{19 L}$ fragment; since they are also formed in the presence of $s c^{H R}$, they probably depend on the gene that is located between the $s c^{H}$ and $s c^{19}$ breakpoints, scute $\beta$. A second group of neurons may form when either the $s c^{19 L}$ or the $s c^{19 R}$ fragment is present. This group is somewhat heterogeneous, in that the contribution of the two regions $\operatorname{sc} \beta$ and sc $\gamma$ is quite variable. Three neurons seem to depend only on $\mathrm{sc} \gamma$; whether or not they represent a separate class is not clear.

All neurons that depend on the $s c^{H L}$ fragment should also be found in the presence of $s c^{19 L}$, since the latter fragment includes the former; this is indeed the case.

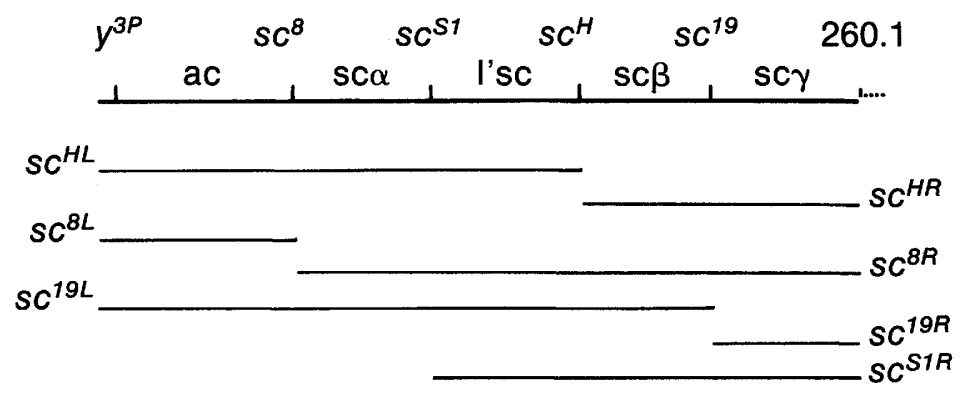

Figure 3. The subdivision of AS-C. The first line shows the location of the breakpoints used to divide the complex in five adjacent regions, according to the molecular map of AS-C (Campuzano et al. 1985). The distance between $\mathrm{y}^{3 P}$ and $s c^{19}$ is approximately $87 \mathrm{~kb}$; the breakpoint of $D f 260.1$ has not yet been mapped. The following lines show the extent of the fragments used in this work. The breakpoint of $s c^{4}$ is very close to that of $s c^{S 1}$ so that $s c^{8 L} S c^{4 R}$ is equivalent to $s c^{8 L} s c^{S 1 R}$, that is, deleted for sc $\alpha(\operatorname{Raffel}$ and $M u l l e r 1940)$. Five transcripts have been identified so far (Campuzano et al. 1985; J. Modolell, pers. comm.): T1a, transcribed from the sc $\gamma$ region, T2 from the sc $\beta$, T3 from l'sc, T4 from sc $\alpha$, and T5 from ac. All but T2 share a very conserved sequence, and are made in the embryo, raising the possibility that T2 is unrelated to AS-C function, and that $\operatorname{sc} \beta$ acts by altering the expression of other AS-C genes. 
The 12 neurons that are missing with both $s c^{H}$ fragments depend entirely on the presence of $s c^{19 L}$, indicating that they require a region of AS-C located between $s c^{8}$ and $s c^{19}$.

\section{Assignment of es neurons to AS-C regions}

The previous results show that different larval sense organs require different regions of AS-C to develop. On the basis of the data in Table 1, one can define four classes of sense organs and neurons that show very distinct requirements for AS-C elements (last line of Table 1, and Fig. 2). The presence of the leftmost region, ac, is sufficient for the development of the first class, which will be called A (for ac). The ac function can to some extent be substituted by sc $\alpha$, and possibly by l'sc, but not by the other genes $\operatorname{sc} \beta$ or sc $\gamma$. The presence of the rightmost region sc $\gamma$ is sufficient for the development of the second class, which will be called $\mathrm{C}$ (for scy). Thus, $\mathrm{C}$ neurons develop whenever sc $\gamma$ is present, even if all other regions are absent. The sc $\gamma$ function can be substituted to a variable extent by $\operatorname{sc} \beta$, but not at all by the leftmost genes ac, sc $\alpha$, or l'sc. A third class, called B (for $\operatorname{sc} \beta$ ) develops whenever $\operatorname{sc} \beta$ is present; however, we cannot say whether the presence of $\operatorname{sc} \beta$ is sufficient, since there exists no genotype where $\operatorname{sc} \beta$ is present and all other AS-C regions are absent. The fourth class, D, requires the integrity of the region between the breakpoints $s c^{8}$ and $s c^{19}$ : these organs will not form when only ac or sc $\gamma$ are present, nor when only part of the central region is present.

On what feature of the sensory system is this division in four classes based? Probably not on temporal differences, since all es neurons differentiate nearly synchronously. Nor is it related to the nature of sense organs: Of the three types of external sense organs found on the body segments of Drosophila larvae, hairs (trichoid sensilla) and papillae (campaniform sensilla) are present in all four classes, and multiply innervated pegs (basiconic sensilla) are present in classes $\mathrm{A}$ and $\mathrm{C}$. What seems to be important is the spatial arrangement of the sense organs within each class, as appears in Figure 2 where we present the subpatterns of neurons and sense organs that correspond to each class. Class A appears to define a basic subpattern that is repeated in all thoracic and abdominal segments (except that the les neuron is duplicated in T1). This subpattern includes one element in the ventralmost cluster, $\mathrm{v}$, two (of which one is multiply innervated) in the next cluster, $v^{\prime}$, one in the lateral cluster, and two in the dorsal cluster. Some elements of this subpattern are also retained in the terminal segment resulting from the fusion of the eighth, the ninth, and the vestigial tenth abdominal segments, most notably the multiply innervated pegs in A8 and A9. Class C contains a second set of segmentally repeated multi-innervated structures as well as several mono-innervated sense organs. Class B comprises a small number of sense organs restricted to the $\mathrm{A}$ and $\mathrm{A} 8$ patterns. Taken together, $B$ and $C$ define a second basic subpattern that is repeated in most body segments. This $B+C$ subpattern includes two sense organs in the $\mathrm{v}$ cluster, two in the 1 cluster, and one in the $\mathrm{d}$ cluster, in addition to the multi-innervated structures mentioned above. Finally, class $D$ includes a few additional sense organs present only in the thoracic segments.

\section{Hidden homologies exposed in bxd mutants}

The hypothesis that class A defines a basic subpattern repeated in most body segments implies that, in spite of the completely different morphology and the more dorsal location, the doubly innervated papilla of the A pattern (p6, arrow in Fig. 2A and 4a,b) and its two neurons ( $v^{\prime} e s 2$ ) is homologous to the ventral peg of the $T$ pattern and its three neurons $\mathrm{v}^{\prime}$ es 3 . We have used the mutation bithoraxoid $(b \times d)$ to reveal this hidden homology. It is known that, in the larva, the mutation bxd makes ventral pegs develop in segments A2-A7 (Lewis 1978 , where these organs are called "ventral pits"). We observed that the presence of such pegs is always associated with the absence of $\mathrm{p} 6$ (but not of the surrounding abdominal sense organs). Furthermore, the location (Fig. $4 \mathrm{~d}-\mathrm{f}$ ) and morphology of these pegs are in many cases intermediate between those of a thoracic peg and those of a p6 papilla, demonstrating the inferred homology between these two structures.

A second case of concealed homology involves the multiply innervated structures of class C. It has already been argued that the thoracic dorsal pegs (multiply innervated dorsal structures in Tl and T; Fig. 2) are homologous to the terminal pegs tl and $\mathrm{t} 2$ (triply innervated structures in A9; Fig. 2) (Sato et al. 1985; Dambly-Chaudière and Ghysen 1986). The fact that the small dorsal hair of the abdominal segments $(\mathrm{h} 3$, arrow in Fig. $2 \mathrm{C}$ and Fig. $5 \mathrm{a}, \mathrm{b}$ ) is doubly innervated by neurons des 2 suggests a possible homology with the dorsal thoracic and terminal pegs. The analysis of $b x d$ larvae shows that $h 3$ is indeed often transformed into a thoracic peg in segments A2-A7. In many cases the transformation affects only the morphology of the sense organ (Fig. 5c) or its location (Fig. 5d); in some cases both the morphology and location are affected (Fig. 5e,f). A partial transformation is observed for about one-half of the organs, and a complete transformation for about one-tenth, in $b x d^{100 /}$ DfP10 larvae.

\section{Discussion}

In adult flies, the development of sense organs according to a defined pattern depends on the complex locus AS-C. Our results show that in the larva, AS-C is required for the development of the different types of external sense organs and their associated neurons (es neurons). In addition, AS-C controls the development of a second category of peripheral neurons, the polydendritic type 2 sensory neurons (nd neurons), which are not associated with specialized sense organs. A third category of peripheral neurons remains unaffected, the ch neurons, which innervate chordotonal organs. Indirect evidence based on the analysis of sensory projections suggests that adult ch neurons are also independent of AS-C (A. Ghysen, unpubl.). Interestingly, L.Y. Jan and Y.N. Jan 

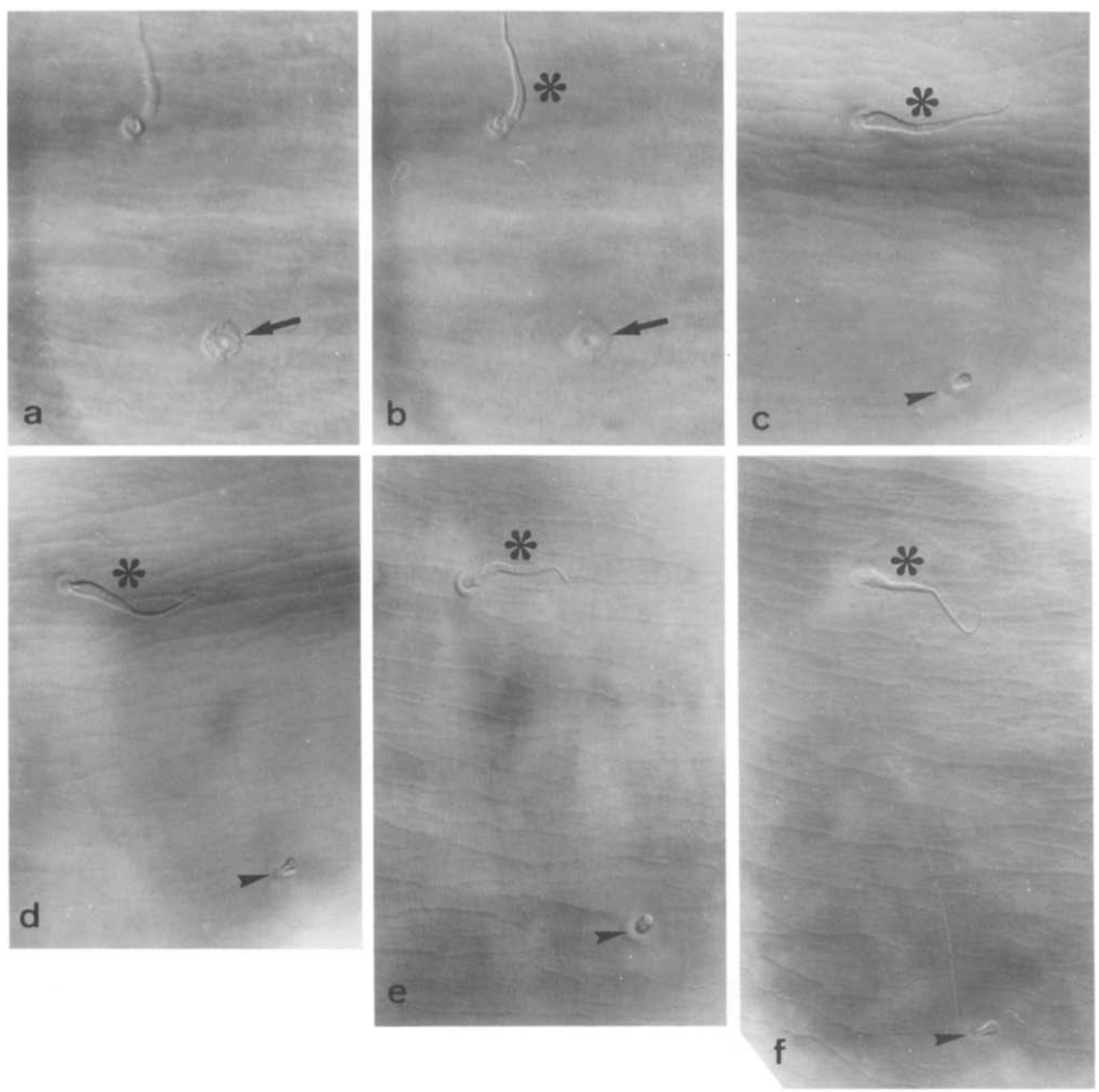

Figure 4. Transformation of the papilla p6 (arrow, Fig. 2a) into a ventral peg. ( $a$ and $b$ ) Normal p6 (arrows) in an abdominal segment of a wild-type larva at two focal levels. The papilla p6, typically surrounded by a ruffled annulus, is in focus with the socket of the lateral hair, hl $\left({ }^{\star}\right)$. When the hair itself is in focus, p6 is slightly out of focus but still clearly detectable $(b) .(c-f)$ Different examples of the transformation of $\mathrm{p} 6$ in $b x d$ larvae. In all cases the lateral hair $\mathrm{h} 1$ is found at its appropriate location $\left({ }^{*}\right)$, while p6 is variably transformed toward a ventral peg (arrowheads). In the most extreme cases the peg is partly buried in a pit (e), as are its thoracic homologs, and is substantially displaced ventrally from the normal p6 location.

have found a gene on the third chromosome that seems specifically required for the development of ch neurons (pers. comm.).

In the adult, a genetic dissection of AS-C has revealed that at least two independent genes are involved in the development of innervated bristles. A similar situation holds in the larva, where our analysis shows that several AS-C genes are required for the development of external sense organs. Even sense organs that are morphologi- cally indistinguishable, such as the various bristles, may depend on different AS-C genes and therefore be genetically diverse. These differences might possibly be used later to control some aspect of neuronal differentiation, such as central pathfinding or targetfinding.

We have identified four classes of sensory elements on the basis of their specific requirements for AS-C function(s). This subdivision is clearly not related to histotype, since each class contains sense organs of at least 

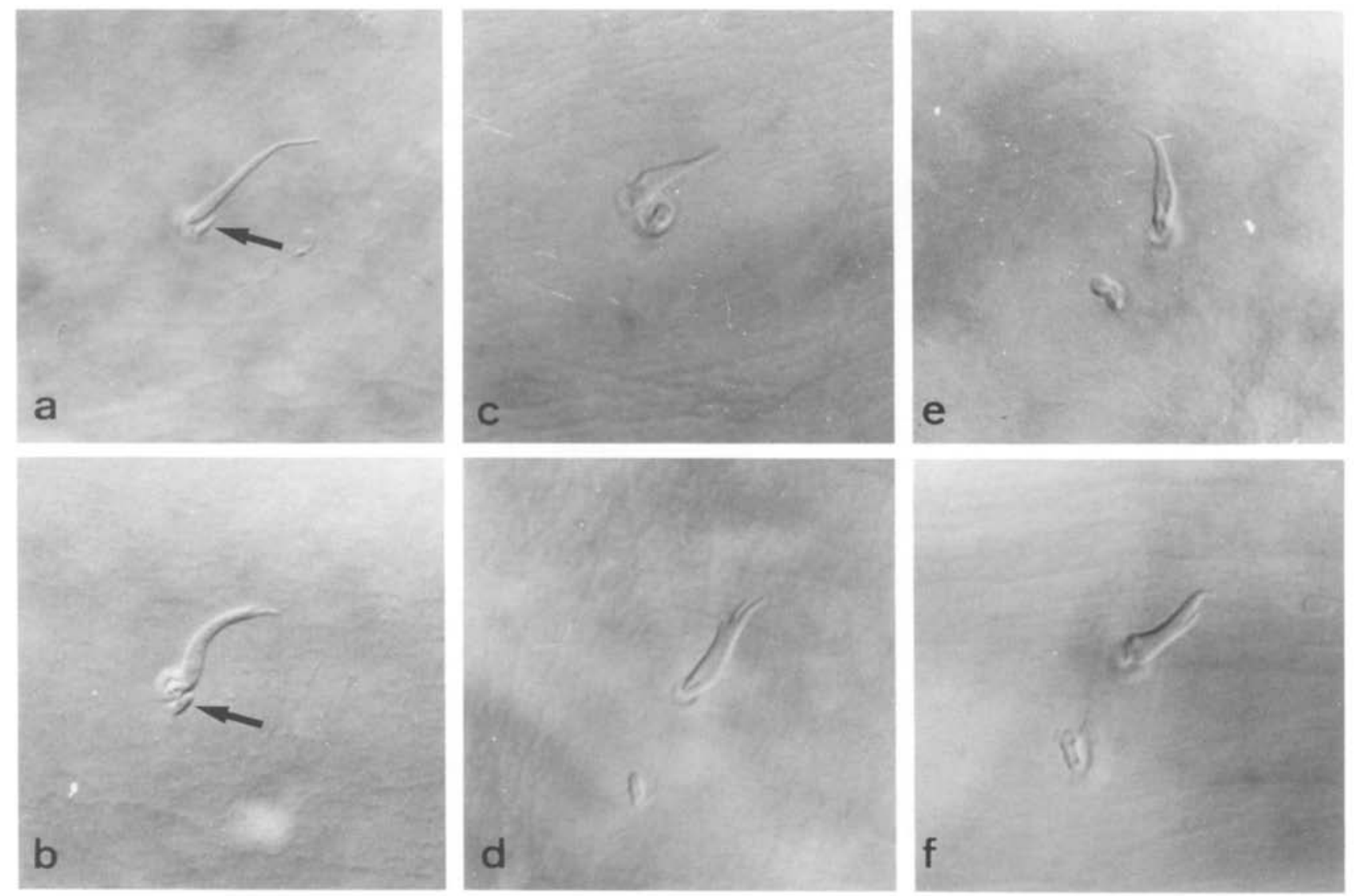

Figure 5. Transformation of the dorsal hair h3 (arrow, Fig. $2 \mathrm{c}$ ) into a peg. ( $a$ and $b$ ) The two dorsal hairs, h3 and h4, in wild-type larvae. The diminutive hair h3 (arrows) is usually sharp, as in $a$, but may occasionally be blunted, as in $b$. $(c-f)$ Different examples of transformation of $\mathrm{h} 3$ in bxd larvac. h3 may be morphologically transformed into a peg (c), or displaced toward the location appropriate for a thoracic peg $(d)$, or both $(e, f)$. In some cases $(c, f)$, the peg is partly buried in a pit.

two and usually all three morphological types. On the other hand, the sense organs assigned to each class show a distinct spatial distribution, so that the four classes define four complementary subpatterns within the larval pattern (Fig. 2). The subpatterns $A$ and $C$, and presumably $\mathrm{B}$ and $\mathrm{D}$ as well, can be formed in the absence of the other three subpatterns, suggesting that the entire pattern of larval sense organs results from the addition of four independent subpatterns. That a given sensory structure may develop normally in the absence of most of its normal neighbors was already observed in the case of adult bristles (Ghysen 1980; Held and Bryant 1984). This independence suggests that if local interactions are involved in the patterning of sense organs (Wigglesworth 1940; Richelle and Ghysen 1979; Garcia-Bellido 1981) these interactions take place among uncommitted epithelial cells, rather than between presumptive sense organs.

Two of the classes, $\mathrm{A}$ and $\mathrm{C}$, define subpatterns that extend along all body segments, from $\mathrm{Tl}$ to $\mathrm{A} 9-10$. In the case of the A subpattern, the precise location of both the neurons and the external sensory structures differ in different segments; for example, the precise position of the singly and multiply innervated elements in the $\mathrm{v}^{\prime}$ cluster differ in the thoracic and anterior abdominal seg- ments. In spite of these differences in the precise position, however, the spatial distribution of sensory elements appears basically similar in all segments (except in the terminal segment, where the massive dorsalization of the pattern might reflect the reshaping of the terminal region of the blastoderm into that of the prospective larva). This basic similarity of the A subpattern in different segments might be fortuitous, or it might indicate that ac defines a spatial arrangement that is segmentally repeated. The analysis of partial segmental transformations in $b x d$ larvae shows that the multiply innervated organs of class A are indeed serially homologous in the thoracic and anterior abdominal segments, even though these organs have very different morphologies and somewhat different location in different segments. The $\mathrm{C}$ subpattern is also suggestive of segmental homologies, between sense organs that differ both in morphology and precise location. Here again the homology was demonstrated by the analysis of $b x d$ larvae.

In addition to the fact that several AS-C genes behave as qualitatively different, in that each corresponds to a specific subset of sense organs, our results also show that some of the genes can substitute for each other, at least partially. For example, the absence of ac is to some extent compensated by the presence of $\operatorname{sc} \alpha$, or even by 
the presence of l'sc. This finding is in remarkable agreement with the recent observation that the proteins encoded for by ac, sc $\alpha$, and l'sc share highly conserved domains (Modolell and Cabrera, pers. comm.). Thus, the functional homology that we have detected is paralleled by a high level of molecular homology, suggesting derivation from a common ancestor. However this molecular homology extends to the sc $\gamma$ product, which does not substitute for ac in the larva. This resembles the situation found in the adult, where the molecularly homologous genes ac and sc $\alpha$ are required for different sets of bristles (Garcia-Bellido and Santamaria 1978) and of campaniform sensilla (Leyns, pers. comm.). Thus, ac and sc $\alpha$ define complementary sets of sense organs in the adult, while in the larva they affect the same subset; and conversely sc $\alpha$ and sc $\gamma$ correspond to different subsets of sense organs in the larva, while they affect the same subset in the adult. These differences in the way AS-C genes combine their effects in the larva and in the adult indicate that there is no simple correspondence between each AS-C gene and a particular subset of sense organs; rather, the results suggest that all AS-C genes perform basically similar operations that are combined in different ways in the generation of the various larval and adult patterns.

Taken together, these results suggest that AS-C is involved in the basic organization of the peripheral nervous system, while both histotype and precise location have to do with the final differentiation, and may depend on other factors.

\section{Conclusion}

In conclusion, our analysis of AS-C shows that the larval pattern of sense organs results from the addition of independent subpatterns that specifically depend on different genes of the complex. Two of the subpatterns, B and $D$, are spatially restricted, one to the thoracic segments and the other to the abdominal segments up to A8. The other two subpatterns, A and C, extend along all body segments, from T1 to A10. Both A and C subpatterns reveal segmental homologies between seemingly quite different neurons in different segments. In two cases where such a hidden homology could be directly demonstrated in $b x d$ larvae, the homologous structures have very different morphologies and somewhat different locations in different segments, making it all but impossible to recognize the homology in the normal larva. This suggests that the essence of a pattern lies in the genetics that generate it, irrespective of the final shape and location of its elements, and that homology can only be ascertained at that level.

\section{Methods}

Larvae or embryos were prepared for microscopic examination of the external sense organs as described in Dambly-Chaudière and Ghysen (1986). Staining of the peripheral neurons of embryos with the monoclonal antibody $21 \mathrm{~A} 4$ was performed as in Ghysen et al. (1986). Ten to 25 embryos or third instar larvae of each genotype were examined. In the case of es neurons, fre- quencies lower than 1 do not necessarily imply that there is an effect on the development of that neuron, since some dendrites are less conspicuous than others and may occasionally remain undetected. In general, es neurons are either completely absent or present and detected in more than $60 \%$ of the cases. There is, however, a clear decrease in the frequency of $C$ neurons when the breakpoint is closer to the scy region: The average frequency for the $16 \mathrm{C}$ neurons is 0.91 in $s C^{8 R}, 0.67$ in $s c^{H R}$, and 0.54 in $s c^{19 R}$. A weaker decrease is observed in the frequency of A neurons when the breakpoint is closer to the ac region: The average for the 21 A neurons is 0.90 in $s c^{19 L}, 0.85$ in $s c^{H L}$, and 0.78 in $s c^{8 L}$.

We observed that hemizygous deficiencies have a more extreme phenotype in females than in males. For example, $D f(1) s c^{H} / D f(1) 260.1$ is more extreme than $D f(1) s c^{H} / Y$. Since the corresponding duplications were all analyzed in male embryos, for example as $D f(1) 260.1 / \mathrm{Y} ; D p(1 ; 4) s c^{H /}+$, we used male embryos for the analysis of the deficiencies as well.

\section{Acknowledgments}

The mutant strains used in this work were obtained from Drs. Campos-Ortega and García-Bellido, and from the Caltech and Umeå stock centers. We thank J. Modolell for communicating results and ideas prior to publication, and A. García-Bellido, R. Thomas, and C. Szpirer for critical reading of the manuscript. This work was supported by FNRS research grants to A.G. and C.D.-C. A.G. is chercheur qualifié of the FNRS.

\section{References}

Bodmer, R. and Y.N. Jan. 1987. Morphological differentiation of the embryonic peripheral neurons in Drosophila. Wilhelm Roux's Arch. Dev. Biol. 196: 69-77.

Campos-Ortega, J.A. and V. Hartenstein. 1985. The embryonic development of Drosophila melanogaster. Springer-Verlag, Berlin, Heidelberg, New York, Toronto.

Campuzano, S., L. Carramolino, C.V. Cabrera, M. Ruíz-Gómez, R. Villares, A. Boronat, and J. Modolell. 1985. Molecular genetics of the achaete-scute gene complex of $D$. melanogaster. Cell 40: 327-338.

Campuzano, S., L. Balcells, R. Villares, L. Carramolino, L. García-Alonso, and J. Modolell. 1986. Excess function Hairy-wing mutations caused by gypsy and copia insertions within structural genes of the achaete-scute complex of Drosophila. Cell 44: 303-312.

Carramolino, L., M. Ruíz-Gómez, M. Guerrero, S. Campuzano, and J. Modolell. 1982. DNA map of mutations at the scute locus of Drosophila melanogaster. EMBO I. 1: 1185-1191.

Dambly-Chaudière, C. and A. Ghysen. 1986. The sense organs in the Drosophila larva and their relation to the embryonic pattern of sensory neurons. Wilhelm Roux's Arch. Dev. Biol. 195: 222-228.

Dubinin, N.P. 1933. Step-allelomorphism in Drosophila melanogaster. J. Genet. 27: 443-464.

García-Alonso, L. and A. García-Bellido. 1986. Genetic studies of Hairy-wing mutations. Wilhelm Roux's Arch. Dev. Biol. 195: $259-264$.

García-Bellido, A. 1979. Genetic analysis of the achaete-scute system of Drosophila melanogaster. Genetics 91: 491-520.

- 1981. From the gene to the pattern: Chaetae differentiation. In Cellular controls in differentiation (ed. C.V. Lloyd and D.A. Rees). Academic Press, New York.

Garcia-Bellido, A. and P. Santamaria. 1978. Developmental analysis of the achaete-scute system of Drosophila melanogaster. Genetics 88: 469-486. 
Ghysen, A. 1980. The projection of sensory neurons in the central nervous system of Drosophila: Choice of the appropriate pathway. Dev. Biol. 78: 521-541.

Ghysen, A., C. Dambly-Chaudière, E. Aceves, L.Y. Jan, and Y.N. Jan. 1986. Sensory neurons and peripheral pathways in Drosophila embryos. Wilhelm Roux's Arch. Dev. Biol. 195: 281-289.

Held, L.I., Jr. and P.J. Bryant. 1984. Cell interactions controlling the formation of bristle patterns in Drosophila. In Pattern formation (ed. G.M. Malacinski and S.V. Bryant). Macmillan, New York.

Jan, L.Y. and Y.N. Jan. 1982. Antibodies to horseradish peroxidase as specific neuronal markers in Drosophila and in grasshopper embryos. Proc. Natl. Acad. Sci. 72: 2700-2704.

Jimenez, F. and J.A. Campos-Ortega. 1979. A region of the Drosophila genome necessary for CNS development. Nature 282: 310-312.

Lewis, E.B. 1978. A gene complex controlling segmentation in Drosophila. Nature 276: 565-570.

Lindsley, D.L. and E.H. Grell. 1968. Genetic variations of Drosophila melanogaster. Carnegie Institute, Washington.

Muller, H.J. and A.A. Prokofyeva. 1935. The individual gene in relation to the chromomere and the chromosome. Proc. Natl. Acad. Sci. 21: 16-26.

Raffel, D. and H.J. Muller. 1940. Position effect and gene divisibility considered in connection with three strikingly similar acute mutations. Genetics 25: 541-583.

Richelle, J. and A. Ghysen. 1979. Determination of sensory bristles and pattern formation in Drosophila. I. A model. Dev. Biol. 70: 418-437.

Sato, T., P.H. Hayes, and R.E. Denell. 1985. Homeosis in Drosophila: Roles and spatial patterns of expression of the $A n$ tennapedia and Sex combs reduced loci in embryogenesis. Dev. Biol. 111: 171-192.

Serebrovsky, A.S. and N.P. Dubinin. 1930. X-ray experiments with Drosophila. J. Hered. 26: 469-478.

Sutton, E. 1943. A cytogenetic study of the yellow-scute region of the X chromosome in Drosophila melanogaster. Genetics 28: $210-217$.

White, K. 1980. Defective neural development in Drosophila melanogaster embryos deficient for the tip of the $\mathrm{X}$ chromosome. Dev. Biol. 80: 332-344.

Wigglesworth, V.B. 1940. Local and general factors in the development of "pattern" in Rhodnius prolixus. I. Exp. Biol. 17: $180-200$. 


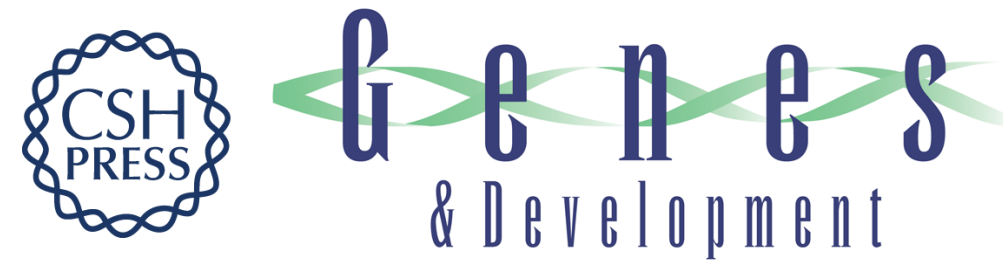

\section{Independent subpatterns of sense organs require independent genes of the achaete-scute complex in Drosophila larvae}

Genes Dev. 1987, 1:

Access the most recent version at doi:10.1101/gad.1.3.297

References This article cites 22 articles, 6 of which can be accessed free at: http://genesdev.cshlp.org/content/1/3/297.full.html\#ref-list-1

License

Email Alerting

Receive free email alerts when new articles cite this article - sign up in the box at the top Service right corner of the article or click here.

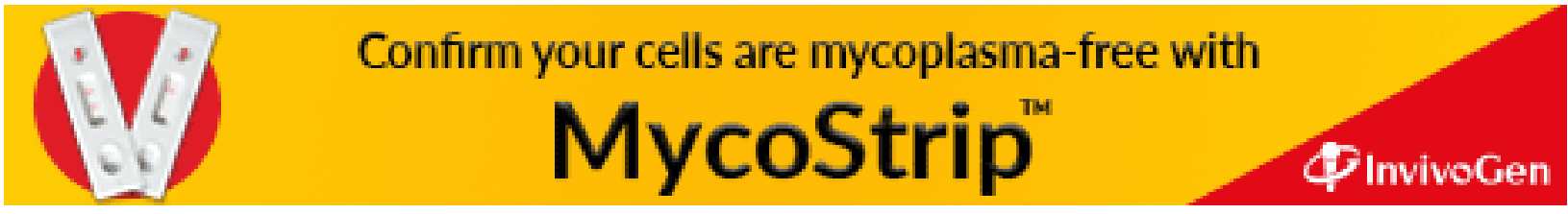

